\title{
Bilateral Adrenal Hemorrhage Secondary to Heparin Induced Thrombocytopenia in a Trauma Patient
}

\section{Yuki Yoshiokaand ${ }^{1}$ and Akihiro Hamanaka ${ }^{2}$}

${ }^{1}$ Department of Emergency Medicine, Taoka Hospital, Japan

${ }^{2}$ Department of Radiology, Awaji Medical Center, Japan

\section{Dear Editor,}

We report a case of a trauma patient with Bilateral Adrenal Hemorrhage (BAH) associated with Heparin Induced Thrombocytopenia (HIT). An 80-year-old man was brought to our hospital after falling from a height of $3 \mathrm{~m}$. His vital signs were stable, and whole body Computed Tomography (CT) showed that he had right multiple rib fractures, hemothorax, and pelvic fracture. The patient subsequently underwent Transcatheter Arterial Embolization (TAE) for the pelvic fracture hemorrhage treatment. Then, the patient was admitted to the intensive care unit and was transfused 2 units of red cell concentrate and 2 units of fresh frozen plasma.

On Day 3, we started with the subcutaneous injection of unfractionated heparin $(15,000$ units per day) to prevent Deep Vein Thrombosis (DVT). On Day 6, the patient vomited and had fever $\left(39^{\circ} \mathrm{C}\right)$ with a confused mental status. His systolic blood pressure suddenly dropped to $90 \mathrm{mmHg}$. Abdominal CT scan demonstrated $\mathrm{BAH}$ (Figure 1) and laboratory findings exhibited a platelet count of 50 $\times 10^{3} \mathrm{~mm}^{-3}$ (which was $160 \times 10^{3} \mathrm{~mm}^{-3} 5$ days earlier). We suspected HIT leading to BAH and stopped heparin injection; we subsequently started anticoagulation therapy with argatrovan (continuous infusion: $0.5 \mu \mathrm{g} / \mathrm{BW} \mathrm{kg} / \mathrm{min}$ ) and checked heparin antibody. As the patient was in a shock status, we diagnosed adrenal insufficiency and started the administration of steroids. Subsequently, the patient gained consciousness, his other symptoms regressed, and his platelet count gradually increased. Later, heparin antibody was found to be positive, and the diagnosis of HIT was confirmed.

\section{Discussion}

Diagnosis of BAH is challenging [1]. For effective treatment, it is important to suspect BAH and take a CT scan, which has excellent diagnostic accuracy in BAH [2]. BAH is a recognized complication of meningococcemia, sepsis, trauma, burn, coagulopathy, and anticoagulation therapy. The symptoms of $\mathrm{BAH}$ are nonspecific and

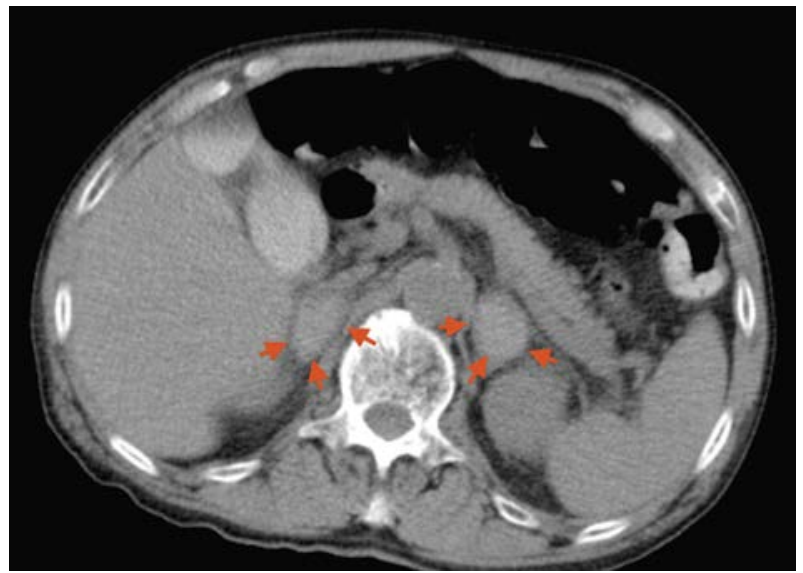

Figure 1: Arrows indicate the bilateral adrenal hemorrhage. variable: they include fever, confused mental status, vomiting, low blood pressure, and abdominal pain. In the case of BAH leading to acute adrenal insufficiency, a delay in diagnosis may be fatal $[3,4]$.

Unfractionated Heparin (UFH) is not recommened to prevent DVT in trauma patients [5]. However, in Japan, Low-MolecularWeight Heparin (LMWH) is not approved to administer to a trauma patient who has not undergone an operation in order to prevent DVT.

HIT causes the venous and arterial thrombosis, leading to various complications, including BAH. As mentioned above, the diagnosis of $\mathrm{BAH}$ is challenging. Therefore, in the clinical course of treatment of trauma patients administered with heparin, BAH associated with HIT should be taken into consideration.

\section{References}

1. Anderson KC, Kuhajda FP, Bell WR (1981) Diagnosis and treatment of anticoagulant-related adrenal hemorrhage. Am J Hematol 11: 379-385.

2. Ling D, Korobkin M, Silverman PM, Dunnick NR (1983) CT demonstration of bilateral adrenal hemorrhage. AJR Am J Roentgenol 141: 307-308.

3. Picolos MK, Nooka A, Davis AB, Raval B, Orlander PR (2007) Bilateral adrena hemorrhage: an overlooked cause of hypotension. J Emerg Med 32: 167-169.

4. Guichelaar MM, Leenen LP, Braams R (2004) Transient adrenocortica insufficiency following traumatic bilateral adrenal hemorrhage. J Trauma 56 1135-1137.

5. Geerts WH, Jay RM, Code KI, Chen E, Szalai JP, et al. (1996) A comparison of low-dose heparin with low-molecular-weight heparin as prophylaxis against venous thromboembolism after major trauma. N Engl J Med 335: 701-707.

*Corresponding author: Yuki Yoshioka, Department of Emergency Medicine, Taoka Hospital, 4-2-2, Bandai-Cho, Tokushima City, Tokushima 770-0941, Japan, Tel: +8188-622-7788; Fax: +81-88-655-3077; E-mail: benguyc@gmail.com

Received November 11, 2014; Accepted December 12, 2014; Published December 15, 2014

Citation: Yoshiokaand Y, Hamanaka A (2014) Bilateral Adrenal Hemorrhage Secondary to Heparin Induced Thrombocytopenia in a Trauma Patient. J Trauma Treat 4: 230. doi:10.4172/2167-1222.1000230

Copyright: (c) 2014 Yoshiokaand Y, et al. This is an open-access article distributed under the terms of the Creative Commons Attribution License, which permits unrestricted use, distribution, and reproduction in any medium, provided the original author and source are credited. 